\title{
Hydraulic Fracture Optimization Model to Maximize Tight Gas Recovery
}

Authors: Md Motiur Rahman and Jorge Salgado Gomes

Petroleum Engineering Department

The Petroleum Institute

Abu Dhabi, UAE

E-mail: mrahman@pi.ac.ae

As the demand for gas increases worldwide, tight and deep unconventional gas reservoirs are becoming the target for development. However, for such reservoirs the conventional approach of simply fracturing the formation in such reservoirs to hydraulically stimulate the well is inadequate. This is because most currently available commercial software may lack proper optimization tools in them and they might not have taken into consideration several key parameters and realistic design constraints in them.

An integrated but constrained model to optimize hydraulic fracture treatment parameters has been developed to maximize gas production as well net present value with minimum treatment cost. A dual objective function has been incorporated to satisfy operator's economic constraints and also to investigate trade-off between two conflicting design objectives. Model can couple both the industry experience and design constraints based on hydraulic fracture mechanics. Realistic design constraints based on field experiences are formulated mathematically and are incorporated in the model. The optimization scheme in the model is driven by an intelligent moving-object algorithm, which is basically developed based on Genetic and Polytope algorithm. Unlike commercial software, important design parameters are included as the free design variables (injection rate, injection time, fracturing fluid viscosity, proppant concentration, etc.) which are randomly varied during optimization. Model has been applied to a hypothetical deeper tight gas formation (a vertical well) to demonstrate its merits. The optimum treatment design indicates a significant incremental production (by 300\% compared to production from non-fractured tight gas well) at lower cost. This model, which needs further refinement for transverse fractures in horizontal well, could also be used to study the potential of the deep UAE gas sands. 